\title{
Spatial evolution of friction of a textured wafer surface
}

\author{
Huaping XIAO $^{1, \mathrm{a}}$, Ke WANG ${ }^{2, \mathrm{a}}$, Grant FOX ${ }^{1, \mathrm{a}}$, Michel BELIN ${ }^{3}$, Julien FONTAINE ${ }^{3}$, Hong LIANG $^{1,2, *}$ \\ ${ }^{1}$ Mechanical Engineering, Texas A\&M University, College Station, Texas 77843, USA \\ ${ }^{2}$ Materials Science and Engineering, Texas A\&M University, College Station, Texas 77843, USA \\ ${ }^{3}$ Ecole Centrale de Lyon/CNRS-UMR 5513, France \\ Received: 3 January 2013 / Revised: 23 February 2013 / Accepted: 27 February 2013 \\ (C) The author(s) 2013. This article is published with open access at Springerlink.com
}

\begin{abstract}
Mechanical failure of integrated circuits and micro-electro-mechanical systems (MEMS) demands new understanding of friction in small devices. In present research, we demonstrated an in situ approach to measure sliding friction of a patterned surface composing multi-materials and structures. The effects of materials and surface morphology on friction and electrical contact resistance were investigated. The material transfer at the interface of dissimilar materials was found to play dominating roles in friction. The current work provides important insights from the fundamentals of friction that benefit the design of new micro-devices.
\end{abstract}

Keywords: micro-electro-mechanical systems (MEMS); patterned wafers; friction; transfer wear; electrical contact resistance

\section{Introduction}

Since Leonardo Da Vinci's first design in tangential force measurement [1-2], the surface friction has been studied for centuries [3-4]. The sliding friction can be evaluated against contact conditions and environments. Existing formulas used to calculate friction are based on static conditions involving a tangential force and applied load over an average size of contact area. In micro-meter length scale, macroscopic friction law is not always applicable [5-6]. With the development of micro system the surface texturing has become important in frictional control. Some natural creatures excel in controlling friction employing specially designed texture to benefit their behavior. Geckos' toes have special shaped seta system which produces high friction enabling geckos to walk and climb easily [7-8]. The stridulation of crickets is produced by the friction induced vibration owing to the textured wing [9-10]. In industrial applications, friction and wear have become the one of the major causes of failure in small devices such as micro-electromechanical systems (MEMS) [11-12]. For MEMS actuators composed of components like gears and hinges, friction and wear

${ }^{\text {a }}$ Xiao, Wang, and Fox contributed equally to this work.

* Corresponding author: Hong LIANG. E-mail: hliang@tamu.edu are inevitable and are important factors for better performance and longer lifespan. In order to improve the quality and the service life of microscale devices, friction and stiction control in interfacial structures is highly desirable [13-14].

In order to understand friction and its impact on surface texture, an in situ method was employed to investigate the tribological properties of the surface during sliding. To achieve precise control, we designed a surface that is textured with two different materials, formed in strips parallel to each other, having different tribological and electrical properties. The electrical contact resistance (ECR) and the coefficient of friction (COF) were evaluated to monitor the change on the surface with evolution of friction. The spatial evolution of friction and its corresponding resistance were expected to reveal the transfer of surface materials due to sliding and assist design of textured surfaces. X-ray photoelectron spectroscopy (XPS) imaging was also conducted to validate the materials transfer.

\section{Experiments}

\subsection{Materials}

The sample is a commercial wafer provided by Semitech Inc. It was chemical-mechanically polished 
and pre-patterned with parallel nickel [15] and silicon dioxide $\left(\mathrm{SiO}_{2}\right)$ stripes. The width of the $\mathrm{Ni}$ strips is in the range of $70-88 \mu \mathrm{m}$ while that of $\mathrm{SiO}_{2}$ strips is in the range of $200-300 \mu \mathrm{m}$. Figure 1 shows a photo of the Ni-Si wafer prior to testing. The hardness of $\mathrm{Si}$ is $13 \mathrm{GPa}$ [16], $\mathrm{SiO}_{2}$ is $9 \mathrm{GPa}$ [17], and $\mathrm{Ni}$ is $600 \mathrm{MPa}$ [18]. The sample was rinsed with acetone for 20 minutes in an ultrasonic cleaner prior to the friction test. To build a conductive circuit for ECR test, the wafer was attached to an aluminum stage using a carbon tape. A droplet of silver paint was added to the wafer edge so that current could get through the conductive Ni strips. A standard bearing ball of AISI 52100 steel with $6 \mathrm{~mm}$ diameter was used to slide against the wafer surface.

\subsection{Tribological testing and characterization}

Friction experiments were conducted using a homemade ball-on-flat tribometer [19] with a linear reciprocal motion. The applied load and the sliding speed are $0.3 \mathrm{~N}$ and $2 \mathrm{~mm} / \mathrm{s}$, respectively, with a track length of $3.5 \mathrm{~mm}$. The coefficient of friction and the resistance between the pin and the disk were recorded simultaneously. The "triboscopical images" revealing the evolution of COF and ECR with increasing number of rubbing cycles was produced [19]. The calculated Hertzian contact pressure is $93.4 \mathrm{MPa}$ on $\mathrm{Ni}$ strips and $57.8 \mathrm{MPa}$ on $\mathrm{SiO}_{2}$. After tribotests, surface profile was measured using a white light interferometer (Zygo Corp., NewView 600).

Chemical analysis was conducted using an XPS (Kratos Axis Ultra imaging). The $\mathrm{Al} \mathrm{K} \alpha$ line with energy of $1486.7 \mathrm{eV}$ was utilized as incident beam and the typical pressure in the spectrometer was around $10^{-7} \mathrm{~Pa}$. Normal incident $\mathrm{X}$-ray was introduced to the

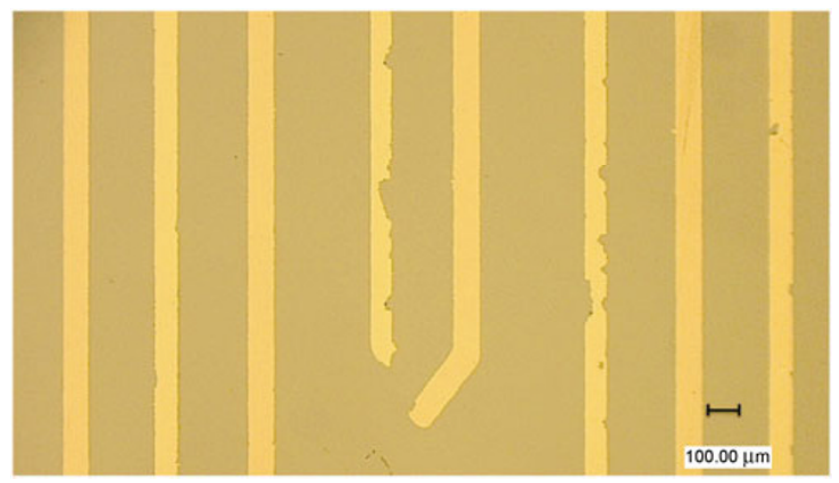

Fig. 1 Photo of Nickel-Silicon wafer at 100× magnification. sample while the angle between the incident direction and the detector direction is $45^{\circ}$. Images were acquired at $853 \mathrm{eV}$ which is the characteristic binding energy (BE) of $\mathrm{Ni}_{2 \mathrm{p} 3 / 2}$.

\section{Results and discussion}

The friction measurement is displayed in a triboscopical image as shown in Fig. 2(a). The $X$-axis is the number of sliding cycles while the $Y$-axis is the position of the sliding pin along the linear track on the disk. The $Y$-axis is composed of 512 elements with a length of approximately $7 \mu \mathrm{m}$ for each element. The intensity of the brightness in Fig. 2(a) represents the value of $\mathrm{COF}$ and brighter zone represents higher COF. The patterns of $\mathrm{Ni}$ and $\mathrm{SiO}_{2}$ stripes on the wafer are clearly distinguished especially in later cycles. A visible change in COF begins around cycle 20. It becomes more evident after cycle 25 . Subsequently, bright strips appear at the interface between $\mathrm{Ni}$ and $\mathrm{SiO}_{2}$ indicating wear initiated at the interface. Between cycles 15 and 40 , the friction continues to rise and varies in a wide area. Soon after the cycle 45 , a high friction zone covering entire sliding track can be distinguished.

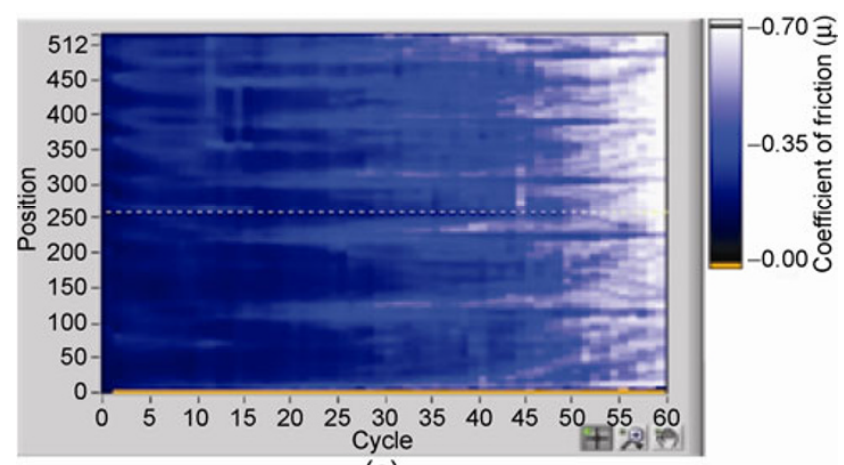

(a)

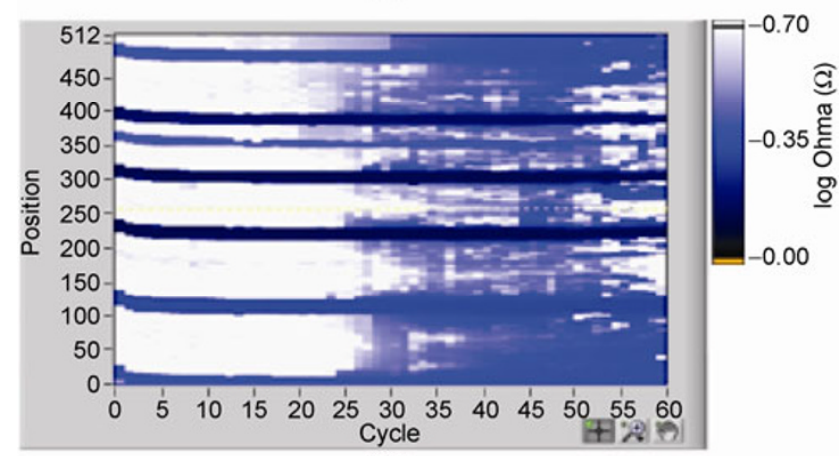

(b)

Fig. 2 (a) Triboscopical image of the coefficient of friction. (b) Triboscopical image of electrical contact resistance. 
The in situ ECR (Ohms, $\Omega$ ) measurement along the wear track was conducted simultaneously. The ECR data were plotted for all cycles on a tricboscopical image, as shown in Fig. 2(b). The intensity of the brightness represents the measured contact resistance. Higher intensity indicates larger contact resistance. The regions with low contact resistance (blue or dark) are $\mathrm{Ni}$ stripes, the bright regions are $\mathrm{SiO}_{2}$ stripes. The difference between pre-patterned $\mathrm{Ni}$ and $\mathrm{SiO}_{2}$ stripes is more evidently demonstrated in the ECR image. In the first 20 cycles, the measured ECR remains stable. The boundaries between $\mathrm{Ni}$ and $\mathrm{SiO}_{2}$ stripes can be clearly distinguished. As shown in Fig. 2(b), the contact resistance begins to drop from the $21^{\text {st }}$ cycle. A transition region starts to form at the interface between $\mathrm{Ni}$ and $\mathrm{SiO}_{2}$ stripes. After the $25^{\text {th }}$ cycle, the decrease of contact resistance is observed in the prior high contact resistance positions, the measured ECR decreasing from $10^{6} \Omega$ to $10^{5} \Omega$ in 10 cycles. Since all other experimental conditions remain the same, this drop could be attributed to the transfer of $\mathrm{Ni}$ during sliding. Conductive $\mathrm{Ni}$ transferred to the non-conductive $\mathrm{SiO}_{2}$ strips greatly reduces the contact resistance in the $\mathrm{SiO}_{2}$ strips. At the $45^{\text {th }}$ cycle, the contact resistance reaches the lowest value, $10^{4} \Omega$. At the end of the test, from the $50^{\text {th }}$ cycle to $60^{\text {th }}$ cycle, the contact resistance is observed to rise. An increase of COF is also identified in Fig. 2(a) at the end of the test suggesting a severe wear at the corresponding positions. $\mathrm{Ni}$ transferred to $\mathrm{SiO}_{2}$ strips could be worn out leading to the increase of ECR.

Furthermore, the average ECR and average COF of each cycle were calculated and plotted in Fig. 3. The results demonstrate the same trend observed in triboscopical image. In the first 20 cycles, both ECR and COF change little with increasing number of cycles. This result suggests that the friction and wear develop slowly at the beginning. This is due to the super finish of the wafer and effects of asperities are limited. After that, the measured ECR starts to decrease fast and reaches the lowest value around the $45^{\text {th }}$ cycle, while the COF starts to increase gradually. With more rubbing cycles, wear steadily progresses leading to the change of the wafer surface morphology. The surface becomes rougher in time. The temperature is high at the contact between the asperities of the wafer surface and the steel ball. The Ni particles scratched away from the Ni strip adhere to the ball due to the high temperature and then travel along the $\mathrm{SiO}_{2}$ strip. In the sling on $\mathrm{SiO}_{2}$ surface, transfer of $\mathrm{Ni}$ from steel ball to $\mathrm{SiO}_{2}$ could be expected. From cycle 20 to cycle 45 , the resistance keeps decreasing which indicates the increase of the conductive film (the transferred $\mathrm{Ni}$ film) on the $\mathrm{SiO}_{2}$ surface. In the last 10 cycles of the test, the measured ECR is observed to raise back, which is accompanied by the increase of COF. The rapid increase in $\mathrm{COF}$ demonstrates the severe wear in this stage. At this stage the Ni film is gradually worn out and the thickness of Ni film decreases while the resistance increases accordingly.

In order to investigate the impact of rubbing cycles on the tribological properties, a multi-sequential test was conducted. The resulting optical micrograph of the wear track is displayed in Fig. 4(a). In region A, B and $C$ the number of rubbing cycle is separately 20 , 45 and 60. A slight wear can be identified from the color difference in region A. The morphology image obtained by white light interferometer confirms this subtle wear as shown in Fig. 4(b). In region B, the sign of wear is evident and surface damage along the wear track can be seen. Figure 4(c) shows the morphology image in this region. As the number of rubbing cycles increases to 60 , the wear track gets much wider and severe surface damage is produced. The Ni strip was worn out as shown in Fig. 4(d). The average surface roughness of the wear track in Fig. $4(\mathrm{~b})$ is $2 \mathrm{~nm}$. And

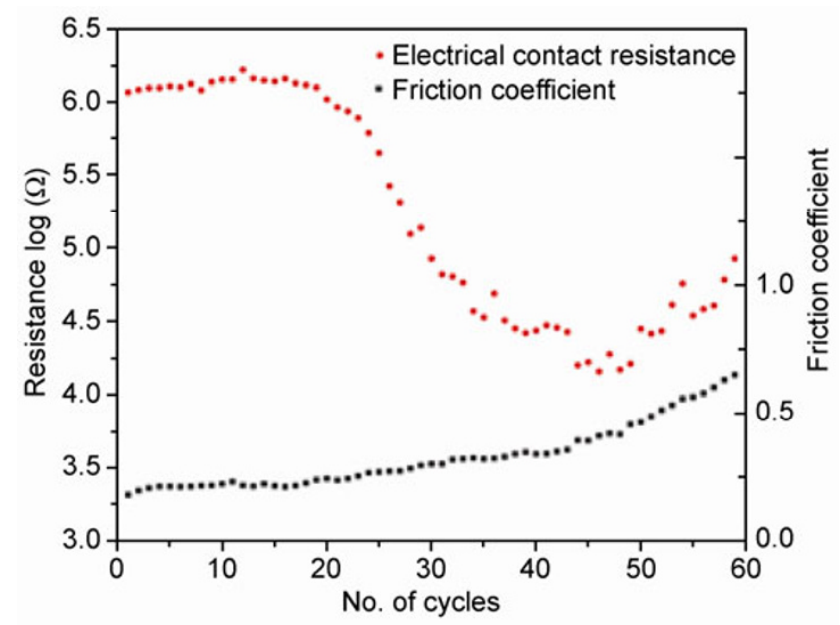

Fig. 3 Average coefficient of friction and electrical contact resistance vs. number of cycles. 

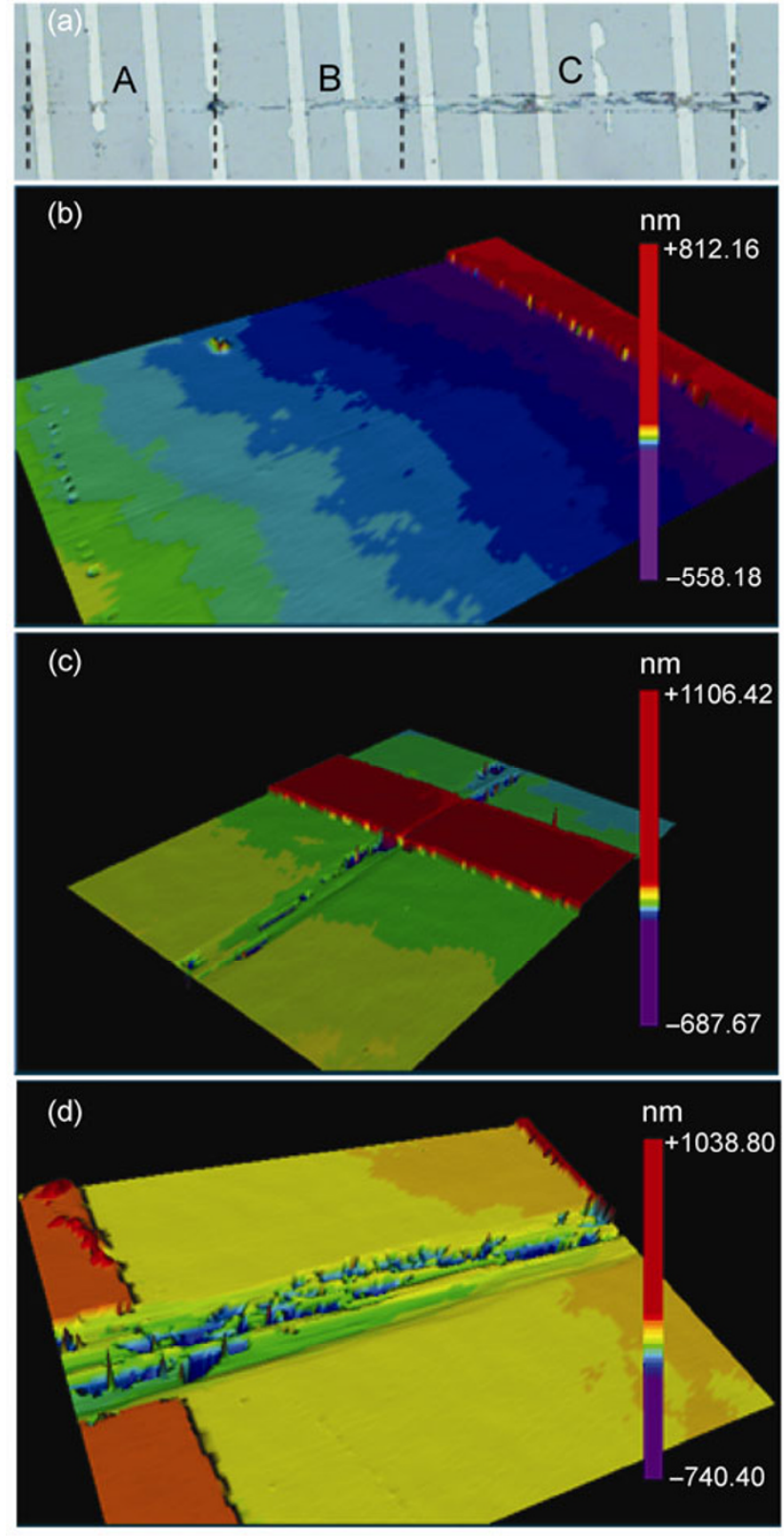

Fig. 4 (a) Optical micrograph of multi-sequential wear track. (b) Morphology of the wear track after 20 cycles. (c) Morphology of the wear track after 45 cycles. (d) Morphology of the wear track after 60 cycles. The total length of wear track is $3.5 \mathrm{~mm}$ in (a). Scanned area is $0.35 \mathrm{~mm} \times 0.26 \mathrm{~mm}$ in (b), (c), and (d).

this value reaches to $24 \mathrm{~nm}$ and $67 \mathrm{~nm}$ in Figs. 4(c) and $4(\mathrm{~d})$. From the morphological characterization, it is evident that the average surface roughness in the wear track region increases significantly with cycles, and more wear damage is introduced on the prepatterned $\mathrm{Ni}$ and $\mathrm{SiO}_{2}$ stripes.

To confirm the material transfer during sliding, XPS imaging was conducted on the wear tracks. Results are shown in Fig. 5. Figure 5(a) shows a worn region with the wear track crossing two pre-patterned $\mathrm{Ni}$ stripes. Figure $5(b)$ is the enlarged image of the inset in Fig. 5(a). The bright zone indicates the existence of $\mathrm{Ni}$. The distribution of $\mathrm{Ni}$ along the wear track can be clearly determined. This confirms the transfer of $\mathrm{Ni}$ from pre-patterned $\mathrm{Ni}$ strips to $\mathrm{SiO}_{2}$ strips during the sliding process. The long traveling distance of the $\mathrm{Ni}$ can be observed from Fig. 5. The brightness in the wear track is between that of the Ni strip and the $\mathrm{SiO}_{2}$ strip, which means the concentration of $\mathrm{Ni}$ in the wear track is between the two pure strips and mechanical mixing of the two elements happens in the wear track. The gradual decrease of ECR from Fig. 3 demonstrates the mechanical mixing of the elements
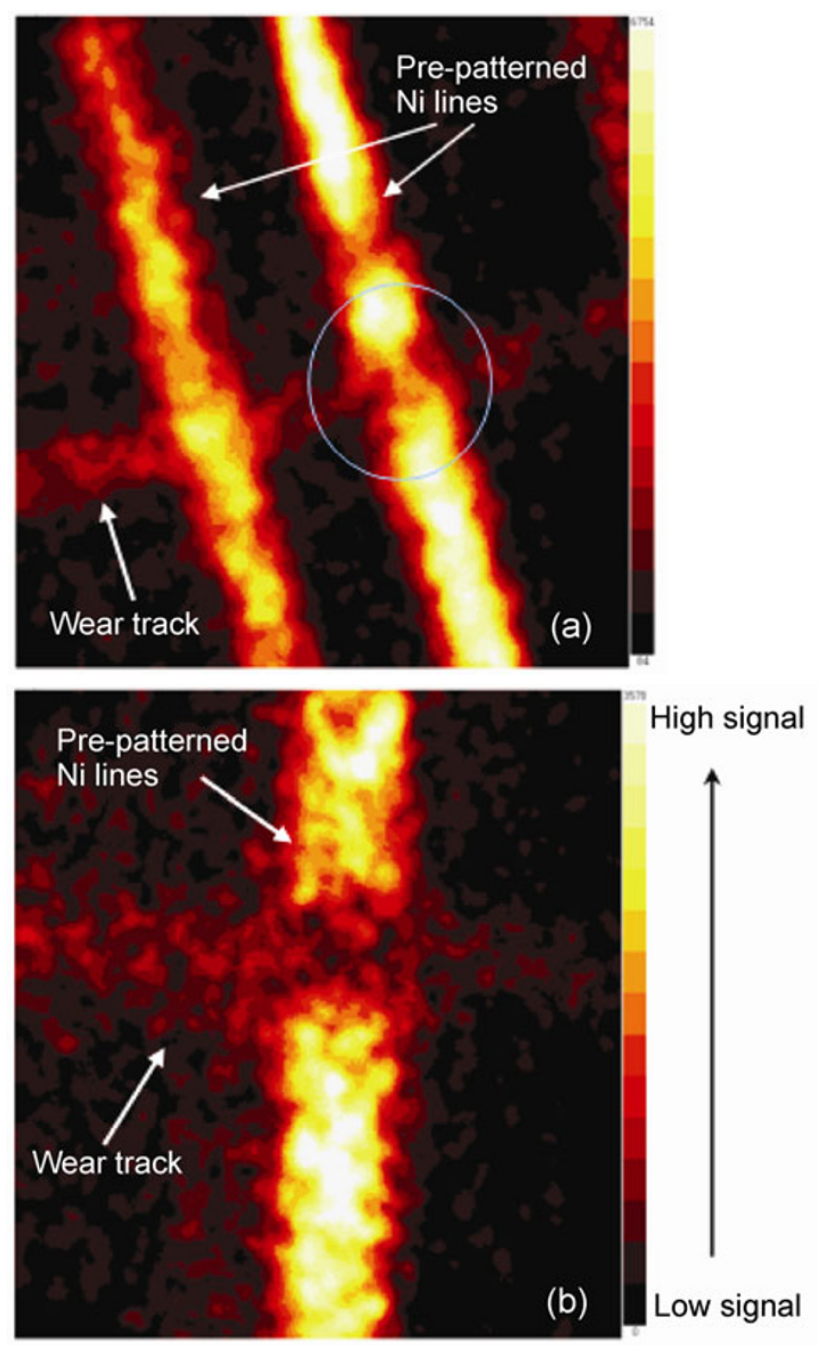

Fig. 5 XPS elemental images $\left(\mathrm{Ni}_{2 \mathrm{p}}\right)$ of tested $\mathrm{Ni}$ patterned $\mathrm{Si}$ wafer. (a) $800 \mu \mathrm{m} \times 800 \mu \mathrm{m}$. (b) Imaging of the circled region in (a), $400 \mu \mathrm{m} \times 400 \mu \mathrm{m}$. 
is an accumulative process during sliding. In this stage, Ni transfer dominates friction. The following increase in ECR suggests the decrease of $\mathrm{Ni}$ concentration and the dominating process is wear of the material.

Based on experimental results, three stages can be distinguished in the cyclic sliding process for the parallel strip structures. In the first stage, the COF is stable and the wear is negligible. In the second stage, the COF gradually increases and evident wear occurs. More importantly, for the parallel strips composed of different materials, the softer material transfers from its own strip to the harder strip. If the electrical conductivities of the two materials have a huge difference, the material transfer will cause a remarkable influence and may cause the failure of the component. In the third stage as shown in Fig. 3, the COF increases progressively and the wear develops accordingly. For most MEMS applications, the components will lose the precision and reliability under severe wear. Based on the fact that the metal-silicon strip structure is widely used in semiconductor industry, the friction and wear should be controlled so that the component can work under the first stage because even small amount of metal transfer will damage the electrical conductivity of the whole component.

\section{Conclusion}

In order to study the kinetics of materials transfer during sliding, friction and electrical contact resistance of a patterned surface were studied. The influence of materials and material transfer on sliding friction was observed in situ. The average COF gradually increased from 0.18 to 0.67 after 60 rubbing cycles. The average ECR reduced from $10^{6} \Omega$ to $10^{4} \Omega$ firstly and then increased to $10^{5} \Omega$ at the end of tests. The XPS results have proven the transfer of materials for a long distance along the wear track and the mechanical mixing is an accumulative process. This study reveals insight into the possible failure mechanisms of sliding of small devices with textured surfaces.

\section{Acknowledgements}

This research was in part sponsored by National
Science Foundation (NSF) (0535578).

Open Access: This article is distributed under the terms of the Creative Commons Attribution Noncommercial License which permits any noncommercial use, distribution, and reproduction in any medium, provided the original author(s) and source are credited.

\section{References}

[1] da Vinci L, Richter I A, Kemp M. Notebooks. Oxford: Oxford University Press, 2008.

[2] da Vinci L. II Codice Atlantico. Milano: Accademia dei Lincei, 1900-1903.

[3] Dowson D. History of Tribology. London and New York: Longman Group Limited, 1979.

[4] Gu X K, Chen M. Shape dependence of slip length on patterned hydrophobic surfaces. Appl Phys Lett 99: 063101 (2011)

[5] Mo Y, Turner K T, Szlufarska I. Friction laws at the nanoscale. Nature 457: 1116-1110 (2009)

[6] Delrio F W, De Bore M, Knapp J A, Reedy Jr E D, Clews P J, Dunn M. The role of van der Waals forces in adhesion of micromachined surfaces. Nature Mater 4: 629-634 (2005)

[7] Tian Y, Pesika N, Zeng H B, Rosenberg K, Zhao B X, McGuiggan P, Autumn K, Israelachvili J. Adhesion and friction in gecko toe attachment and detachment. Proc Nat Acad Sci USA 103: 19320-19325 (2006)

[8] Persson B N J, Gorb S. On the mechanism of adhesion in biological systems. J Chem Phys 118: 7614-7621 (2003)

[9] Bailey W J. The mechanics of stridulation in Bush Crickets (Tettigonioidea, Orthoptera): I. The tegminal generator. $J$ Exp Biol 52: 495-505 (1970)

[10] Bennet-Clark H C, Bailey W J. Ticking of the clockwork cricket: The role of the escapement mechanism. J Exp Biol 205: 613-625 (2002)

[11] Patton S T, Zabinski J S. Failure mechanisms of a MEMS actuator in very high vacuum. Tribol Int 35: 373-379 (2002)

[12] van Spengen W M, Puers R, De Volf I. The prediction of stiction failures in MEMS. IEEE Transction on Device and Material Reliability 3: 167-172 (2003)

[13] Liu F, Laborianet I, Bush B, Roper C S, Carraro C, Maboudian R. In situ studies of interfacial contact evolution via a two-axis deflecting cantilever microinstrument. Appl Phys Lett 95: 131902 (2009)

[14] Ansari N, Ashurst W R. Single-crystal-silicon-based microinstrument to study friction and wear at MEMS sidewall interfaces. J Micromech Microeng 22: 025008 (2012) 
[15] Montealegre Z F, Windmill J F C, Morris G K, Robert D. Mechanical phase shifters for coherent acoustic radiation in the stridulating wings of crickets: the plectrum mechanism. J Exp Biol 212: 257-269 (2009)

[16] Bhushan B, Li X. Micromechanical and tribological characterization of doped single-crystal silicon and polysilicon films for microelectromechanical systems devices. J Mater Res 12: 54-63 (1997)

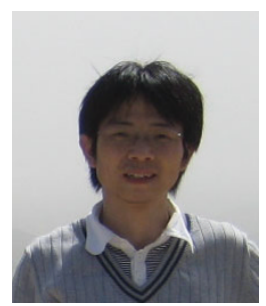

Huaping XIAO. Ph. D. candidate at Department of Mechanical Engineering, Texas A\&M University (TAMU), USA. He obtained his Bachelor degree in 2006 from Tianjin University and Master's degree in

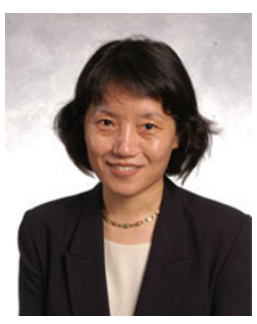

Hong LIANG. Professor at Texas A\&M University, USA. Dr. Liang conducted her postdoc research at National Institute of Technology (NIST) after obtaining her Ph. D. from Stevens Institute of Technology. She is a fellow of American Society
[17] Stone D, LaFontaine W R, Alexopoulos P, Wu T-W, Li $\mathrm{C}-\mathrm{Y}$. An investigation of hardness and adhesion of sputterdeposited aluminum on silicon by utilizing acontinuous indentation test. J Mater Res 3: 141-147 (1988)

[18] Sale J M. Wear resistance of silicon carbide composite coatings. Met Prog 4: 44-45 (1979)

[19] Belin M, Martin J M. Triboscopy, a new approach to surface degradations of thin films. Wear 156: 151-160 (1992)

2011 from Tsinghua University. Since 2011, he has been at TAMU working with Dr. Liang. His research areas include lubrication properties and wear behaviors under high pressure and bio-related tribological systems.

of Mechanical Engineers (ASME) and a fellow of Society of Tribologists and Lubrication Engineers (STLE). Dr. Liang has extensive experience in academia, government laboratories, and industry. She has maintained long-standing interests and activity in tribology, surface science, chemical-mechanical planarization, and nanomanufacturing. 